\title{
Specific features of current and emerging mobile health apps: user views among people with and without mental health problems
}

\author{
Louise K. Thornton ${ }^{1}$, Frances J. Kay-Lambkin ${ }^{1,2}$ \\ ${ }^{1}$ National Drug and Alcohol Research Centre, The University of New South Wales, Sydney, Australia; ${ }^{2}$ School of Medicine and Public Health, The \\ University of Newcastle, Newcastle, Australia \\ Contributions: (I) Conception and design: All authors; (II) Administrative support: LK Thornton; (III) Provision of study materials or patients: All \\ authors; (IV) Collection and assembly of data: LK Thornton; (V) Data analysis and interpretation: All authors; (VI) Manuscript writing: All authors; \\ (VII) Final approval of manuscript: All authors. \\ Correspondence to: Louise K. Thornton. National Drug and Alcohol Research Centre, 22-32 King Street, Randwick, NSW 2031, Australia. \\ Email: L.Thornton@unsw.edu.au.
}

\begin{abstract}
Background: Despite widespread development of mobile health apps, there is a paucity of research investigating user views of apps and their features, particularly among people with mental health problems. This study aimed to gain an understanding of the acceptability of specific features of current and emerging mobile health apps among people with and without mental health problems.

Methods: Adults living in Australia were recruited to complete a self-report questionnaire regarding demographic characteristics, mental health, technology use, and attitudes regarding specific features of mobile health apps.

Results: A total of 284 participants were recruited, including $53 \%$ with a history of mental illness. Few concerns were reported with mobile app features (mean $=1.8$ features; SD $=2.19$ ) and statistical analyses found that age, gender, income, rurality, mental health status and technology use did not affect the expression of these concerns. Key issues identified included privacy and security of personal information, apps acting automatically, sharing personal information and the invasive nature of some features.

Conclusions: These results suggest that mental illness may not be a barrier to widespread use of mobile technologies for health purposes. It is hoped that this research will assist developers and clinicians to develop and integrate mobile health apps into everyday care more effectively.
\end{abstract}

Keywords: Smartphones; mobile health; mobile applications; mental disorder

Received: 12 March 2018; Accepted: 15 November 2018; Published: 06 December 2018.

doi: 10.21037/mhealth.2018.11.04

View this article at: http://dx.doi.org/10.21037/mhealth.2018.11.04

\section{Introduction}

Mobile devices, such as smartphones, have become an integral part of life for many people (1). In 2015, only $12 \%$ of the global population reported that they did not own a cellphone of any kind and $43 \%$ reported owning a smartphone (2). Among richer countries, rates of smartphone ownership are much higher (e.g., South Korea, 88\%; Australia: 77\%; US, 72\%; UK, 68\%) (2). For many people their smartphone is an essential part of their daily lives, and for a growing number of people, and particularly among disadvantaged populations (2), smartphones are also the only way in which they can access a telephone service or the Internet $(2,3)$.

The utility of smartphones to help to improve physical and mental health, and wellbeing, has been widely recognized $(4,5)$. The increasing ubiquity of these devices, and their portable nature, means the reach of services can be greatly increased, while many of their features can 
be used in ways which may serve to improve outcomes and engagement with physical and mental health care interventions. These technologies offer opportunities to build social support, access multimedia features, easily monitor progress towards behaviour change, and offer ongoing personalized care for a fraction of the cost of traditional care (6-9). Their potential to improve existing psychological services and administer psychosocial treatments, is of particular interest (10-12) given the welldocumented difficulties associated with effectively engaging people with mental disorders with health care $(13,14)$.

In recognition of this potential, many thousands of mobile applications (or apps) addressing a wide range of physical and mental health issues have been developed and are available directly to consumers. However, reviews consistently show that the vast majority of these apps have not been developed by health professionals or academics and few are evidence based or have been evaluated in any way $(5,12,15)$. Among the few apps that have been evaluated, findings indicate that mobile applications can be effective in improving physical activity, weight loss, alcohol use, smoking cessation, and mental health problems including depression and anxiety $(4,5,15)$. However much of this data has been generated by small studies of specific applications (12). There is also a paucity of research investigating user views of mobile health apps, and opinions and attitudes regarding specific features of these technologies, especially context sensing features (features that use the sensor built into smartphones such as the GPS, microphone, camera, accelerometer to compute things about the users) $(5,12)$. However, in order to develop health apps that are likely to be acceptable to a target population and to effectively integrated these tools within everyday clinical and public health practice, it is important to have a good understanding of the population of interest's views regarding the features of health apps.

Dennison et al. (12) conducted some of the only research to investigate perspectives of health apps and some of the specific features of current and emerging applications. They conducted focus groups with 18 young adults in the UK and assessed their experiences using health apps, interests and feelings about the features, and capabilities of these apps. They found that participants had some interest in using health apps, and that the ability to track behaviours and goals, receive advice and information "on the go" were highly valued. Social media and context sensing features were less valued. Some participants raised concerns regarding privacy and security of their health data and felt that context-sensing features in particular felt intrusive and would discourage them from using an application. Participants were also skeptical whether phones could sense context accurately enough to actually be useful.

It is unclear if people with mental health problems are likely to hold similar opinions to the general population regarding the features and capabilities of health apps. Previous research has suggested that some characteristics of mental disorders (such as paranoia, cognitive deficits, and social withdrawal) may present significant barriers to the wide scale use of smartphones for health purposes in these populations $(9,16,17)$.

The current study aimed to gain an understanding of the acceptability of specific features of current and emerging mobile health apps among people with and without mental health problems, and the nature of any concerns they might have with using mobile health apps containing these features. It also set out to determine what, if any, demographic and mental health factors might influence people's attitudes towards these features.

\section{Methods}

\section{Participants and procedure}

Eligible participants were aged 18 years and over, and currently living in Australia. Participants were recruited Australia-wide, using paid and unpaid advertisements on Facebook, twitter and via the Hunter Medical Research Institute's (HMRI) research register. The HMRI research register is a database of people based in the Hunter region of New South Wales, Australia who are interested in contributing to medical research.

A paid Facebook advertising campaign was run between July and September 2015 and June and August 2016, in which study advertisements were targeted to appear on the Facebook profiles of users aged over 18 years who listed their location as Australia. As a key aim of this research was to compare people with and without mental health problems, a number of the advertisements and posts were also targeted to appear on the Facebook profiles of users who expressed an interest in Facebook pages related to mental health (e.g., Reachout.com Australia, Headspace, Lifeline, Beyond Blue). The administrators of relevant online communities were also contacted and asked to post the study advertisement on their website and/or social media pages.

Advertisements and posts promoted that participants 
could go into the draw to win an iPad by completing an online (or paper-based) self-report survey, that the survey constituted research, and that this research was being conducted by researchers at the National Drug and Alcohol Research Centre at the University of New South Wales. The researchers also shared the study advertisements on their personal social media profiles.

Potential participants who clicked the study advertisements or posts were taken to an online eligibility screener, information statement and consent form. If they chose to participate, they were then directed to the selfreport questionnaire that was hosted by the online survey program Fluid Surveys.

Two hundred members of the HMRI research register were mailed an invitation to participate in the current study by the HMRI research register coordinator. The researchers were then sent the contact details of members who responded to this invitation. Interested members were mailed a questionnaire pack that included a paper copy of the information statement, the self-report questionnaire and a reply-paid envelope. Return of the completed survey was taken as consent to participate.

\section{Measures}

Participants indicated if they had ever been diagnosed with a mental illness. Current psychological distress was assessed using the Patient Health Questionnaire (PHQ4) (18). The self-report questionnaire also included items regarding demographic characteristics, mobile phone and Internet access and use, openness to using mobile and Internet technologies for health purposes and attitudes regarding a range of features of current and emerging mobile technologies.

Participants indicated how frequently they used the Internet and their mobile-phone, as well as if they had ever used the Internet or their mobile phone to access health information or treatment.

A number of features of current and emerging health apps were described to participants and they were asked to indicate if they thought each feature would be useful to them, if they would be interested in using a health app with that feature and if they had any problems or concerns regarding that feature. The list of features described was adapted from Dennison et al.'s (12) qualitative investigation of users' views and experiences of mobile health apps and included: setting goals, advice and information, tools to monitor behaviour, reminders, showing progress towards a goal, social networking, sharing on social networks, sensing where you are, sensing what you are doing, and sensing how you feel (see Supplementary file 1).

\section{Data analysis}

Data analysis was conducted using STATA and SPSS. Participants' postcodes were used to determine the rurality of where they were living using the Accessibility/ Remoteness Index of Australia (ARIA) (19). ARIA provides a remoteness, or accessibility, value for every location in Australia, allowing researchers to identify if participants lived in major cities, inner regional areas, outer regional areas, or remote areas of Australia.

The proportion of participants expressing an interest or concern in each of the specific features listed was calculated. The specific features were subjected to exploratory factor analysis to determine any relationships between the features and group them accordingly. Based on this analysis, the specific features were grouped into four overarching types of features: basic features (setting goals, advice and information, reminders); monitoring features (tools to monitor behavior, showing progress towards a goal); social networking features (social networking, sharing on social networks); and context sensing features (sensing where you are, sensing what you are doing, sensing how you feel). Binary variables for each of these feature types were created with participants' scored as "yes-have problems or concerns" if they reported having problems or concerns with at least one of the specific features of that type. A series of four logistic regressions were conducted to assess the impact of a number of demographic and technology use factors on the likelihood that participants would report that they had a problem or concern with each of the four types of mobile health app features. The models contained 11 variables (age, gender, income, employment, education, rurality, history of mental disorder, current psychological distress as measured by the $\mathrm{PhQ} 4$, frequency of internet use, previous use of the internet for health concerns and previous use of a mobile phone for health concerns).

The total number of specific features a participant reported having problems or concerns with was also summed and a negative binomial regression conducted to assess the impact of the factors listed above on the likelihood that respondents would report more problems or concerns with the features of mobile health apps.

A content analysis was conducted using the free response of participants regarding their concerns with the features 
Table 1 Participant characteristics $(\mathrm{n}=284)$

\begin{tabular}{|c|c|}
\hline Participant characteristics & Value \\
\hline \multicolumn{2}{|l|}{ Age, years } \\
\hline Range & $18-77$ \\
\hline Mean (SD) & $30.64(14.49)$ \\
\hline \multicolumn{2}{|l|}{ Gender, \% } \\
\hline Male & 28.8 \\
\hline Female & 68.7 \\
\hline Other & 2.8 \\
\hline Born in Australia, \% & 84.9 \\
\hline ATSI, \% & 2.8 \\
\hline LGBTIQ, \% & 19.1 \\
\hline \multicolumn{2}{|l|}{ Marital status, \% } \\
\hline Married, de facto or living with a partner & 50.7 \\
\hline Separated or divorced & 4.6 \\
\hline Never married or single & 42.6 \\
\hline Widowed & 2.1 \\
\hline English not spoken as first language at home, \% & 12.4 \\
\hline \multicolumn{2}{|l|}{ Highest education, \% } \\
\hline High school years $7-10$ & 6.0 \\
\hline High school years 11-12 & 21.8 \\
\hline TAFE or other trade qualification & 22.5 \\
\hline University degree & 49.6 \\
\hline \multicolumn{2}{|l|}{ Personal income each week, \% } \\
\hline$<\$ 100$ & 11.3 \\
\hline$\$ 100-299$ & 14.8 \\
\hline$\$ 300-599$ & 22.3 \\
\hline$\$ 600-999$ & 23.7 \\
\hline$>\$ 1,000$ & 20.8 \\
\hline Prefer not to answer & 7.1 \\
\hline \multicolumn{2}{|l|}{ Employment status, \% } \\
\hline Employed (casual, full- or part-time) & 55.1 \\
\hline Unemployed & 4.2 \\
\hline Student & 20.5 \\
\hline Retired & 7.1 \\
\hline Permanently unable to work & 1.8 \\
\hline Home duties & 4.2 \\
\hline Other & 7.1 \\
\hline
\end{tabular}

Table 1 (continued)
Table 1 (continued)

\begin{tabular}{lc}
\hline Participant characteristics & Value \\
\hline Rurality, \% & 79.4 \\
Major cities & 10.0 \\
Inner regional areas & 9.6 \\
Outer regional areas & 1.1 \\
Remote areas & 53.3 \\
History of mental illness, \% & 28.1 \\
Current psychological distress & 71.9 \\
\hline Moderate to severe & \\
None to mild & \\
\hline ATSI, Aboriginal and Torres Strait Islander; LGBTIQ, Lesbian, \\
gay, Bisexual, Transexual, Intersex, Queer; TAFE, Technical and \\
Further Education. \\
$\quad$ listed or general concerns with mobile health apps. \\
Results
\end{tabular}

A total of 722 people accessed the online survey, of whom 444 consented and 334 were eligible to participate in the study. Eight-five participants stopped completing the online questionnaire part way through, leaving a total of 249 participants who were recruited online with sufficient data to be involved in subsequent analysis.

Of the 200 members of the HMRI register invited, 38 indicated their interest to participate and 35 returned the completed questionnaire. In total, 284 participants were included in the current study.

\section{Participant characteristics}

The typical participant was aged 30 years (mean age $=30.64$, $\mathrm{SD}=14.49)$, female $(68.7 \%)$, employed $(55.1 \%)$ and lived in a major city (79.4\%). Approximately half of participants reported a history of mental illness (53.3\%), however most reported no (or mild) current psychological distress (71.9\%). See Table 1 for a full list of participant characteristics.

Participants reported frequent internet use (daily or more frequent use reported by $98 \%$ of participants, see Table 2) and almost all had access to a smartphone (96.1\%). Almost all participants had used the Internet to access health information or treatment (91.5\%), four fifths (78.2\%) had used their mobile phone to access health information or 
treatment in the past.

\section{Participant attitudes towards specific features}

\section{Quantitative results}

Table 3 presents results regarding participants' attitudes towards specific features of current and emerging mobile health applications. Basic and monitoring features that are available in many existing apps were perceived to be potentially useful and of interest to most participants (65-79\%). Context sensing features were endorsed less frequently, approximately half of participants thought these

Table 2 Internet and mobile phone access and use

\begin{tabular}{lc}
\hline Internet and mobile phone access \& use & $\%$ \\
\hline Frequency of internet use & 41.9 \\
Several times an hour & 15.5 \\
Every hour/once an hour & 40.5 \\
Once to several times a day & 2.1 \\
Once to several times a week & 0 \\
Less than once a week & 100 \\
Own or have access to a mobile phone & 96.1 \\
Own or have access to a "smartphone" & 91.5 \\
$\begin{array}{l}\text { Previous use of the Internet to access health } \\
\text { information or treatment }\end{array}$ & 78.2 \\
$\begin{array}{l}\text { Previous use of a mobile phone to access health } \\
\text { information or treatment }\end{array}$
\end{tabular}

features would be useful and of interest to them (49-57\%). The least frequently endorsed features were those regarding social networking, particularly sharing on social networks (20-43\%).

Similarly, fewer participants reported having problems or concerns with basic and monitoring features (6-14\%). About a quarter of participants reported problems or concerns with social networking (20-31\%) and context sensing features $(25-27 \%)$. The feature that the greatest proportion of participants were concerned by was sharing their information on social networks (30.5\%). Overall, participants had problems or concerns with very few features (mean $=1.8$ features, $S D=2.19$ ). Forty-six percent of participants reported that they did not have any problems or concerns with any of the features described and only $6.7 \%$ of participants reported problems or concerns with 5 or more features.

Logistic regressions found that none of the demographic, mental health or technology use variables included in the models uniquely predicted the likelihood that participants had problems or concerns with the types of features described (all $\mathrm{P}$ values $>0.01$ ). The one exception was employment status with regards to problems or concerns with monitoring features, where employment status was found to have a unique effect over and above the effects of the other factors included in the regression. People who reported working home duties were 42.2 times more likely to have problems or concerns with monitoring features than participants who were employed outside of the home $(\mathrm{P}<0.005)$.

Negative binominal regression assessed the impact of

Table 3 Participants' views regarding specific features of mobile health apps

\begin{tabular}{llccc}
\hline Feature type & Specific feature & Perceived to be useful (\%) & Interested in using (\%) & Problems or concerns (\%) \\
\hline Basic & Setting Goals & 73.24 & 72.63 & 11.07 \\
& Advice and information & 75.18 & 75.46 & 14.12 \\
& Reminders & 66.43 & 7.47 & 12.50 \\
Monitoring & Tools to monitor behaviour & 72.08 & 71.22 & 9.16 \\
& Showing progress towards a goal & 78.57 & 41.99 & 20.11 \\
Social networking & Social networking & 43.01 & 22.79 & 30.50 \\
& Sharing on social networks & 20.43 & 49.26 & 26.54 \\
Context sensing & Sensing where you are & 50.36 & 53.31 & 25.38 \\
& Sensing what you are doing & 55.87 & 57.35 & 24.71 \\
\hline
\end{tabular}


Table 4 Key themes raised regarding concerns with specific mobile app features

\begin{tabular}{ll}
\hline Feature types & Themes \\
\hline All features & Concerns with health apps acting automatically \\
Importance of being able to switch off or customize reminders, data collection and information sharing \\
features \\
Importance of information provided being accurate and evidence based \\
Importance of information offered being tailored to the user \\
Concern that some users would use advice offered within an app instead of seeking appropriate medical advice \\
Dislike being monitored by an app \\
Concern that showing failure to progress towards a goal might be disheartening \\
Nonitoring features & Concern that users may not know who would see their shared information \\
Social networking features & Socially unacceptable to share health information via social media \\
Risk of receiving negative feedback from others as a result of sharing information \\
Concern that talking to others with similar health issues may be detrimental to own progress
\end{tabular}

the factors listed above on the total number of features participants reported having problems or concerns with. While the full model was statistically significant $\left[\chi^{2}(29)=54.281, P=0.003\right]$, education status was the only unique predictor of total number of concerns $\left[\chi^{2}(3)=18.197, \mathrm{P}<0.001\right]$. Participants who reported their highest qualification to be a Technical and Further Education (TAFE) or other trade qualification did not differ from those who reported leaving school at year 10 (approximately 16 years of age) or earlier in the total number of features they had problems or concerns with. In contrast, participants who left school after years 11 or 12 , or completed a university degree, reported problems or concerns with significantly more features than those who had finished school in year 10 or earlier. The incident rate of problems or concerns with specific features among year 11 or 12 graduates was 5.98 times higher $\left[\chi^{2}(1)=7.519\right.$, $\mathrm{P}=0.006]$, and 5.135 times higher for University graduates $\left[\chi^{2}(1)=7.076, P=0.008\right]$, than among those who finished their schooling in year 10 or earlier.

\section{Qualitative results}

The key themes raised by participants regarding their concerns with the various mobile app features studied are summarized in Table 4. Across all types of features, participants raised concerns with health apps acting automatically (e.g., sending reminders and automatically recording or sharing information about their use), and described the importance of being able to switch off or customize these features. Example comments include:

"Must not automatically share info-only when instructed to do so"

"Depends on how much control you have over the reminders. It could get annoying if they go off at inconvenient times, or too often"

"[Context sensing features]-would need to be optional or only at certain times/locations."

Other key themes that emerged for each of the 4 types of features are discussed below.

\section{Basic features}

The main concern of participants regarding basic features was that any advice or information offered in an app should be up-to-date, from a credible source and evidence based. It was also important that it should be easy to determine that these criteria had been met.

"How trustworthy the information was, people might receive money to promote certain things."

"The advice and information would have to come from a credible source and be always up to date, reflecting new research and breakthroughs."

"How do you know if the advice/information is appropriatel correct?"

Participants also discussed the need for any information or advice offered within a health app to be tailored to the 
user, rather than providing general information. This was particularly important for physical health-related conditions (weight, diet, exercise). A number of participants also raised the concern that users would use information or advice offered within an app instead of seeking medical advice from a suitable health professional.

"I think it's important to keep in-mind that two people of a similar height/build \&/or circumstance, may have very different health goals for themselves. Theses apps need to be personalized \& flexible, in order to cater to the needs of its users."

"Some users might take this as solid fact or diagnosis when they should actually see a doctor."

"Advice is generally too general, which largely can't be helped, but regularly results in misdiagnosis."

\section{Monitoring features}

The key concern mentioned by participants regarding monitoring features was that they did not like the idea of being 'watched' or monitored by an app. Example comments include:

"Apps that overtly or covertly track personal information make me uncomfortable and I would generally not use them and remove them."

"Very much just Orwellian concerns of controlling the public."

"Depends on how it's done, don't like the feeling of been watched and tracked."

A few participants expressed concerns that a feature showing progress towards a goal might be disheartening if they failed to meet their goals. They also highlighted the need to support people to meet their goals, as well as just showing their progress.

"If I'm not making progress it can actually be discouraging."

"What happens if I fall bebind in achieving my goal? Support, reinforcement \& encouragement is essential in assisting me to keep on, keeping on."

\section{Social networking features}

When discussing social networking features the major problem or concern raised by participants was that they did not want to, or do not feel comfortable, sharing health information publically. Some participants described that sharing health information with just friends and family or within a private group might be acceptable, but many people were uncomfortable sharing their health information via their usual social media accounts with anyone. For example,

"I may not want something personal, like mental health issues or weight loss shared with other people."

"If I could share information within private groups on FB say, might be interested. But would not be interested to share that kind of information through my public profile."

"I don't want my friends to know what I'm doing bealth wise."

"Really not at all keen on integration with social networksI very rarely post anything on Facebook and the like and would really dislike an app to be associated with my social media accounts as I consider my health info to be private."

Participants raised the concern about losing control of the data and not knowing who would see the information.

"There are always concerns about social networking, mainly the question of who will see the information."

In addition, participants expressed that they felt it was socially unacceptable to share health information via social media, particularly if they were perceived to be sharing information that was too personal or too frequently ("oversharing"):

"Coming across as overly concerned with my weight/health isn't something I'd like. Others may not understand why I have certain goals. Also I'm not one to publish my life on social media."

"Super annoying when people log every little thing. Should be used for when people get a new personal best or smash a goal."

"There's this thing called 'over sharing' and I don't want to tell everyone what I'm doing for every single second of the day."

Participants were also concerned that sharing health information on social networks could open them up to negative feedback from others or "trolling".

"It just makes me feel fat and uncomfortable posting things like that on social media, it doesn't matter if you've lost 400 kilos people will still bully you."

"Whilst social media can have positive side effects it is always open to other people's comments, opinions and trolling which may counteract any positive benefits or support of goals by friends."

Finally, participants discussed that connecting with others with similar health or mental health issues via social networks may not be helpful to achieving their own health goals, and even potentially harmful.

"Focusing on depression and suicide is catching so I'm not sure I'd want to meet up with people who are draining when I'm trying to not think about my own issues."

"I don't think comparing your goals is a great idea."

"Misinformed advice may be taken seriously."

\section{Context sensing features}

By far the most common concern regarding context sensing features was privacy. Participants expressed particular concern over who the information collected by an app would be shared with. Participants were also concerned about the security of the data collected by an app containing these types of features, pointing out this type of data could 
make them quite vulnerable to cyber-attack. For example,

"Would be okay depending on if this information is shared with third parties."

"I don't need, or want, a third-party app learning what I do or where I go either regularly, or on occasion. I feel it's a breach of privacy."

"Violation of privacy. What can it do? Report your location to authorities? Potentially useful but for many it's a nuisance."

"I'd be a bit wary of how this information is used by the app developer as it raises privacy concerns for me. Also would be concerned if this info got into the wrong hands (e.g., hackers being able to see where users of an app are, safety/security issues there)."

Participants also described generally being uncomfortable with these types of features describing them as being "invasive" or "creepy".

"General concern with smartphones being 'too smart' integrating all that info just doesn't sit right with me."

"This feels creepy an intrusive."

"Bit big brothery."

Many participants were also unsure of how mobile health apps could accurately sense information about them and were skeptical if this was even possible. This was particularly the case for sensing emotions and feelings. Some participants pointed out the potential harm if these types of features were not accurate. Comments included:

"My partner of 4 years can't do that sometimes, how is my dang phone going to?!"

"I don't think we have the technology to do this effectively yet."

"Margin for error. I don't like that a program can presume bow I am feeling during a particular period without having any context."

"Amazing if accurate but disastrous if not."

\section{Discussion}

This research is the first to examine users' views of a range of specific features of mobile health apps within a large sample $(n=248)$; the first to do so in an Australian context; and the first to examine if a variety of demographic and mental health factors may influence these views and attitudes. Additionally, the integration of qualitative methods allows for a more detailed and rich description of participant experiences, which is useful when investigating new and complex areas such as users views of mobile health apps. As such this paper adds considerably to our understanding of which features of mobile health apps might be most acceptable to end-users and provides important insights that could be used by developers, researchers and clinicians to improve the development of mobile health apps and their integration into everyday clinical care and public health practice.

This study found that few participant characteristics systematically influenced participants' attitudes towards specific features. Participants of different ages and genders did not differ in the total number, or types, of features that they had problems or concerns with. Importantly, mental health status did not appear to effect willingness to use mobile apps for health, indicating that mental illness may not be a barrier to widespread use of mobile technologies for health purposes among this population. Participants with higher levels of education were found to have problems or concerns with a greater number of features of mobile health apps. This finding may suggest that more educated users of mobile health apps are more cautious consumers of technology. While this research found that the majority of participants did not have any problems of concerns with the features described, between 6 and 30 percent of participants did report problems of concerns with the described features. When designing mobile health apps, or attempting to disseminate them or integrate them into clinical care, it is important that we respect and address these concerns. This could potentially be achieved by including this information in publicly available background or descriptions of the app (akin to a "caveat emptor" tab in the description of all apps), and/or be the focus of discussions with clinicians seeking to integrate the use of mobile app technology into an episode of care. A discussion about prior positive and negative experiences of mobile phone apps could occur, and be used to address concerns about privacy, access, and monitoring features raised by end users.

In general, the results of the current study suggest we need to ensure high levels of privacy and data security, while also considering the burden using these apps places on people, the social acceptability of various actions (e.g., sharing information on social networks), and accepting that users are likely to have individual preferences regarding these issues. In addition to programming mobile phone apps with these high levels of integrity, there is a clear need to communicate these standards to the general public (the consumer market for these apps). Potential users need information regarding the intent and rationale for asking people to use some of the features of mobile health apps.

The findings of the current study are closely aligned to those of Dennison et al.'s (12) focus group research, in which greater acceptance and interest in basic and monitoring features was reported compared to social networking and 
context sensing features. Participants in their study also expressed concerns regarding privacy and viewed context sensing features with scepticism and to be intrusive.

In the current study, while basic features were frequently endorsed by participants and the sample reported few concerns with these types of features in general, the results highlight a number of issues developers may wish to consider when integrating these types of features into their mobile health apps. When developing an app, our results suggest that it is important to ensure that information provided is evidence based, and that this is clear to users. This could involve including references, published research, and clear statements about the developer affiliations. Where possible and appropriate, developers should build in the capability to tailor advice and information to the individual user, and allow any in-app reminders to be customizable. With regards to tools to monitor behaviour, it may also be important to make the purpose of any automatic monitoring or tracking clear to users, as well as providing them with the option to customize or turn off all or some of the monitoring features.

If wanting to incorporate social networking or context sensing features within in an app, these results suggests it is critical to ensure that strong data security and privacy structures are in place, and to inform users exactly how their information will be used, shared and protected. It may also be necessary to take the extra time to help potential users to understand why these types of features could be useful in helping them to improve their health outcomes, and provide opportunities for them to engage with these particular features anonymously (or without linkage to other social networking profiles). Developers could consider instead setting up closed or secret groups within social networking sites or using completely separate social networking sites, while respecting that some people still may not want to share their information.

A key limitation of the current research is that participants were primarily recruited online (via social networking sites), and as such their views regarding the use of technology for health purposes may not be representative of the broader population and may not capture the views of non-social media users. However, it should be noted that the majority of Australian adults are users of social media (20). Similarly, participants were required to be currently living in Australia so results may not be generalizable to other contexts.

Despite these limitations, this research has important implications for clinicians, who should feel more comfortable recommending mobile health apps to clients with mental health issues. It is also hoped that this research will assist developers to make more informed decisions regarding the types of features they might incorporate in their mobile health tools and assist those attempting to integrate mobile health apps into everyday clinical care to do so by providing insights regarding the issues or concerns end-users might have.

\section{Acknowledgements}

This work was supported by Dr. Thornton's University of New South Wales Vice-Chancellor's Post-doctoral fellowship.

\section{Footnote}

Conflicts of Interest: The authors have no conflicts of interest to declare.

Disclaimer: The funder of this study (the University of New South Wales) did not have any role in the study design, collection, analysis and interpretation of the data, writing of the report or the decision to submit the report for publication. The authors had full access to all of the data in this study.

\section{References}

1. Barratt MJ, Lenton S. Beyound recruitment? Participatry online research with people who use drugs. International Journal of Internet Research Ethics 2010;3:69-86.

2. Pew Research Center. Smartphone ownership and internet usage continues to climb in emerging economies. US: 2016. Available online: http://www.pewglobal. org/2016/02/22/smartphone-ownership-and-internetusage-continues-to-climb-in-emerging-economies/

3. ACMA. Australians get mobile. Australian Communication and Media Authority, 2015. Available online: https:// www.acma.gov.au/theACMA/engage-blogs/engage-blogs/ Research-snapshots/Australians-get-mobile

4. Payne HE, Lister C, West JH, et al. Behavioral functionality of mobile apps in health interventions: a systematic review of the literature. JMIR Mhealth Uhealth 2015;3:e20.

5. Donker T, Petrie K, Proudfoot J, et al. Smartphones for smarter delivery of mental health programs: a systematic review. J Med Internet Res 2013;15:e247.

6. Brouwer W, Kroeze W, Crutzen R, et al. Which 
intervention characteristics are related to more exposure to internet-delivered healthy lifestyle promotion interventions? A systematic review. J Med Internet Res 2011;13:e2.

7. Mishra GD, Hockey R, Powers J, et al. Recruitment via the Internet and social networking sites: the 1989-1995 cohort of the Australian Longitudinal Study on Women's Health. J Med Internet Res 2014;16:e279.

8. Mitchell JW, Petroll AE. Patterns of HIV and STI testing among MSM couples in the US. Sex Transm Dis 2012;39: 871-6.

9. Firth J, Cotter J, Torous J, et al. Mobile Phone Ownership and Endorsement of "mHealth" Among People With Psychosis: A Meta-analysis of Cross-sectional Studies. Schizophr Bull 2016;42:448-55.

10. Miyagi E, Motoki Y, Asai-Sato M, et al. Web-based recruiting for a survey on knowledge and awareness of cervical cancer prevention among young women living in Kanagawa prefecture, Japan. Int J Gynecol Cancer 2014;24:1347-55.

11. Proudfoot J. The future is in our hands: the role of mobile phones in the prevention and management of mental disorders. Aust N Z J Psychiatry 2013;47:111-3.

12. Dennison L, Morrison L, Conway G, et al. Opportunities and challenges for smartphone applications in supporting health behavior change: qualitative study. J Med Internet Res 2013;15:e86.

13. Judd F, Jackson H, Komiti A, et al. Help-seeking by rural residents for mental health problems: the importance of

\section{doi: 10.21037/mhealth.2018.11.04}

Cite this article as: Thornton LK, Kay-Lambkin FJ. Specific features of current and emerging mobile health apps: user views among people with and without mental health problems. mHealth 2018;4:56. agrarian values. Aust N Z J Psychiatry 2006;40:769-76.

14. Ben-Zeev D, Davis KE, Kaiser S, et al. Mobile technologies among people with serious mental illness: opportunities for future services. Adm Policy Ment Health 2013;40:340-3.

15. Thornton L, Quinn C, Birrell L, et al. Free smoking cessation mobile apps available in Australia: a quality review and content analysis. Aust N Z J Public Health 2017;41:625-30.

16. Sanghara H, Kravariti E, Jakobsen H, et al. Using short message services in mental health services: assessing feasibility. Ment Health Rev (Brighton) 2010;15:28-33.

17. Daker-White G, Rogers A. What is the potential for social networks and support to enhance future telehealth interventions for people with a diagnosis of schizophrenia: a critical interpretive synthesis. BMC Psychiatry 2013;13:279.

18. Kroenke K, Spitzer RL, Williams JB, et al. An ultra-brief screening scale for anxiety and depression: the PHQ-4. Psychosomatics 2009;50:613-21.

19. National Centre for Social Applications of GIS. Accessibility/Remoteness Index of Australia (ARIA) National Centre for Social Applications of GIS (GISCA) and Australian Department of Health and Ageing; 1998 [cited 2016 September]. Available online: http://www. adelaide.edu.au/apmrc/research/projects/category/aria.html

20. Sensis. Sensis Social Media Report 2016. Available online: https://www.sensis.com.au/ 


\section{Supplementary file 1}

Attitudes regarding features of current and emerging mobile technologies for health applications [adapted from Dennison et al. (12)]

1. Do you think any of the following features would be useful for you?

\begin{tabular}{|c|c|c|}
\hline Features & Yes & No \\
\hline Setting goals: some health apps can help you to set goals or plans, e.g., set a weight goal & 1 & 2 \\
\hline Advice and information: some health apps provide access to information, advice or tips & 1 & 2 \\
\hline $\begin{array}{l}\text { Tools to monitor behavior: some health apps provide ways of tracking things to do with health behavior, } \\
\text { e.g., calorie counting food and exercise logs }\end{array}$ & 1 & 2 \\
\hline $\begin{array}{l}\text { Reminders: some health apps beep, vibrate or send you messages or notifications to remind you to do } \\
\text { certain things, e.g., exercise }\end{array}$ & 1 & 2 \\
\hline $\begin{array}{l}\text { Showing progress towards a goal: some health apps can show you your progress towards meeting } \\
\text { health related goals }\end{array}$ & 1 & 2 \\
\hline $\begin{array}{l}\text { Social networking: some health apps allow you to connect and communicate with other people with the } \\
\text { same health problem or issue }\end{array}$ & 1 & 2 \\
\hline $\begin{array}{l}\text { Sharing or social networks: some health apps have the option to share what you are doing with your } \\
\text { online social networks, e.g., Facebook }\end{array}$ & 1 & 2 \\
\hline $\begin{array}{l}\text { Sensing where you are: some health apps use the GPS (location) sensors in your phone to do something } \\
\text { based on your location. These types of apps may be able to notice that you are in a risky/vulnerable } \\
\text { location in terms of making good health choices or sense opportunities for health behaviours }\end{array}$ & 1 & 2 \\
\hline $\begin{array}{l}\text { Sensing what you are doing: some health apps can combine lots of sources of data to "sense" what you } \\
\text { are doing. They might use GPS, accelerometer (whether your phone has been moving around), time of } \\
\text { day and other bits of data to determine where you are and what you are doing. Health apps might use } \\
\text { this information to give some specific health advice or reminder }\end{array}$ & 1 & 2 \\
\hline $\begin{array}{l}\text { Sensing how you feel: in the future some health apps might be able to use data on your phone } \\
\text { (e.g., GPS sensors to determine if you are at home or at work, time or day, presence of other phones } \\
\text { nearby, whether you have recently received or made calls or tests, the tone and volume of your voice) to } \\
\text { make a prediction about what your mood is like. Based on this these types of apps might offer specific } \\
\text { health advice or information }\end{array}$ & 1 & 2 \\
\hline
\end{tabular}

2. Would you be interested in using a health app with the following features?

\begin{tabular}{|c|c|c|}
\hline Features & Yes & No \\
\hline Setting goals: some health apps can help you to set goals or plans, e.g., set a weight goal & 1 & 2 \\
\hline Advice and information: some health apps provide access to information, advice or tips & 1 & 2 \\
\hline $\begin{array}{l}\text { Tools to monitor behavior: some health apps provide ways of tracking things to do with health behavior, } \\
\text { e.g., calorie counting food and exercise logs }\end{array}$ & 1 & 2 \\
\hline $\begin{array}{l}\text { Reminders: Some health apps beep, vibrate or send you messages or notifications to remind you to do } \\
\text { certain things e.g. exercise }\end{array}$ & 1 & 2 \\
\hline $\begin{array}{l}\text { Showing progress towards a goal: Some health apps can show you your progress towards } \\
\text { meeting health related goals }\end{array}$ & 1 & 2 \\
\hline $\begin{array}{l}\text { Social networking: Some health apps allow you to connect and communicate with other people with the } \\
\text { same health problem or issue }\end{array}$ & 1 & 2 \\
\hline $\begin{array}{l}\text { Sharing or social networks: Some health apps have the option to share what you are doing with your } \\
\text { online social networks, e.g., Facebook }\end{array}$ & 1 & 2 \\
\hline $\begin{array}{l}\text { Sensing where you are: Some health apps use the GPS (location) sensors in your phone to do something } \\
\text { based on your location. These types of apps may be able to notice that you are in a risky/vulnerable } \\
\text { location in terms of making good health choices or sense opportunities for health behaviours }\end{array}$ & 1 & 2 \\
\hline $\begin{array}{l}\text { Sensing what you are doing: some health apps can combine lots of sources of data to "sense" what you } \\
\text { are doing. They might use GPS, accelerometer (whether your phone has been moving around), time of } \\
\text { day and other bits of data to determine where you are and what you are doing. Health apps might } \\
\text { use this information to give some specific health advice or reminder }\end{array}$ & 1 & 2 \\
\hline $\begin{array}{l}\text { Sensing how you feel: In the future some health apps might be able to use data on your phone } \\
\text { (e.g., GPS sensors to determine if you are at home or at work, time or day, presence of other phones } \\
\text { nearby, whether you have recently received or made calls or tests, the tone and volume of your voice) to } \\
\text { make a prediction about what your mood is like. Based on this these types of apps might offer specific } \\
\text { health advice or information }\end{array}$ & 1 & 2 \\
\hline
\end{tabular}

\section{Do you have any problems of concerns about the following features?}

\begin{tabular}{lccc}
\hline Feature & Yes & No & If yes, please specify \\
\hline Setting goals & 1 & 2 & \\
Advice and information & 1 & 2 & 2 \\
Tools to monitor behavior & 1 & 2 \\
Reminders & 1 & 2 \\
Showing progress towards a goal & 1 & 2 \\
Social networking & 1 & 2 \\
Sharing or social networks & 1 & 2 \\
Sensing where you are & 1 & 2 \\
Sensing what you are & 1 & 2 \\
Sensing how you feel & 1 & \\
\hline
\end{tabular}

4. Do you have any other general concerns with any of the features listed above, or other features of smartphone apps that you may have heard of? 